\title{
The association between body mass index class and coronavirus disease 2019 outcomes
}

\author{
Abdallah Al-Salameh $\mathbb{1}^{1,2}$ - Jean-Philippe Lanoix ${ }^{3}$ Youssef Bennis ${ }^{4}$ - Claire Andrejak ${ }^{5}$ - Etienne Brochot ${ }^{6}$. \\ Guillaume Deschasse ${ }^{7}$. Hervé Dupont ${ }^{8}$. Vincent Goeb $\mathbb{D}^{9} \cdot$ Maité Jaureguy $^{10}$. Sylvie Lion ${ }^{11}$ - Julien Maizel ${ }^{12}$. \\ Julien Moyet ${ }^{7} \cdot$ Benoit Vaysse $^{13} \cdot$ Rachel Desailloud $^{1,2} \cdot$ Olivier Ganry $^{14} \cdot$ Jean-Luc Schmit ${ }^{3}$. Jean-Daniel Lalau ${ }^{1,2}$
}

Received: 3 June 2020 / Revised: 6 October 2020 / Accepted: 5 November 2020 / Published online: 21 November 2020

(c) Springer Nature Limited 2020

\begin{abstract}
Background/Objectives A growing body of data suggests that obesity influences coronavirus disease 2019 (COVID-19). Our study's primary objective was to assess the association between body mass index (BMI) categories and critical forms of COVID-19. Subjects/Methods Data on consecutive adult patients hospitalized with laboratory-confirmed COVID-19 at Amiens University Hospital (Amiens, France) were extracted retrospectively. The association between BMI categories and the composite primary endpoint (admission to the intensive care unit or death) was probed in a logistic regression analysis.

Results In total, 433 patients were included, and BMI data were available for 329: 20 were underweight (6.1\%), 95 have a normal weight (28.9\%), 90 were overweight $(27.4 \%)$, and 124 were obese $(37.7 \%)$. The BMI category was associated with the primary endpoint in the fully adjusted model; the odds ratio (OR) [95\% confidence interval (CI)] for overweight and obesity were respectively 1.58 [0.77-3.24] and 2.58 [1.28-5.31]. The ORs [95\% CI] for ICU admission were similar for overweight (3.16 [1.29-8.06]) and obesity (3.05 [1.25-7.82]) in the fully adjusted model. The unadjusted ORs for death were similar in all BMI categories while obesity only was associated with higher risk after adjustment.

Conclusions Our results suggest that overweight (and not only obesity) is associated with ICU admission, but overweight is not associated with death.
\end{abstract}

Supplementary information The online version of this article (https:// doi.org/10.1038/s41366-020-00721-1) contains supplementary material, which is available to authorized users.

Abdallah Al-Salameh

al-salameh.abdallah@chu-amiens.fr

1 Department of Endocrinology, Diabetes Mellitus and Nutrition, Amiens University Hospital, Amiens, France

2 PériTox $=$ UMR_I 01, University of Picardie Jules Verne, Amiens, France

3 Department of Infectious Diseases, Amiens University Hospital, Amiens, France

4 Laboratory of Clinical Pharmacology, Amiens University Hospital, Amiens, France

5 Department of Pulmonary diseases, Amiens University Hospital, Amiens, France

6 Laboratory of Virology, Amiens University Hospital, Amiens, France

7 Department of Geriatrics, Amiens University Hospital, Amiens, France

\section{Introduction}

A growing body of evidence suggests that obesity is among the most common conditions associated with coronavirus

8 Surgical Intensive Care Unit, Amiens University Hospital, Amiens, France

9 Department of Rheumatology, Amiens University Hospital, Amiens, France

10 Department of Nephrology, Amiens University Hospital, Amiens, France

11 Department of Orthopedics and Traumatology, Amiens University Hospital, Amiens, France

12 Medical Intensive Care Unit, Amiens University Hospital, Amiens, France

13 Department of Medical Informatics, Amiens University Hospital, Amiens, France

14 Department of Epidemiology, Amiens University Hospital, Amiens, France 
disease 19 (COVID-19). Indeed, $\approx 40 \%$ of patients hospitalized with COVID-19 are obese [1, 2]. In particular, morbid obesity appears to be associated with a severe clinical course [3-9]. There are several mechanistic explanations for this association. First, obesity is associated with respiratory dysfunction, which may predispose obese individuals to lung infections [10, 11]. Second, obesity (especially abdominal obesity) is accompanied by low-grade inflammation, which might modify the immune response to COVID-19. Third, obese individuals frequently have other cardiometabolic conditions that increase the risk of infection by severe acute respiratory syndrome coronavirus 2 (SARS-CoV-2) $[6,12,13]$. It is therefore essential to gain a better understanding of the relationship between obesity and COVID-19. Here, we report on the association between body mass index (BMI) categories and critical forms of COVID-19 (i.e., those requiring admission to the intensive care unit (ICU) or leading to death).

\section{Patients and methods}

We retrospectively extracted data on consecutive adult patients hospitalized with laboratory-confirmed COVID-19 at Amiens University Hospital (Amiens, France). Confirmed COVID-19 was defined as a positive result on the reverse-transcriptase polymerase chain-reaction assay of nasopharyngeal swab specimens. The inclusion criteria were a confirmed diagnosis of COVID-19 and inpatient admission to Amiens University Hospital. The main exclusion criteria were opposition to data collection by the patient or his/her legal guardian and age under 18 .

The extracted data included demographics, risk factors, medical history, medications of special concern, main clinical data, routine laboratory results, and outcomes. All data were double-checked by the first author, who vouches for the data's accuracy. The study was conducted in compliance with good clinical practice. In line with the French legislation on a retrospective analysis of data gathered during routine clinical practice, the study was registered with the French National Data Protection Commission (Commission nationale de l'informatique et des libertés; reference: PI2020_843_0051). Patients who expressed their opposition to data collection were excluded. Patient confidentiality was protected by the assignment of an anonymous identifier to each enrolled participant. The identifier was attributed when the data were extracted, and only anonymized data were analyzed.

The primary endpoint was a composite of ICU admission and death. The secondary endpoints were the components of the primary endpoint. Of note, some deaths occurred in the ICU and they were recorded for both secondary endpoints. Other endpoints were also recorded: a requirement for mechanical ventilation, a record of acute respiratory distress syndrome (ARDS, according to the Berlin criteria [14]) on the ICU discharge note, acute coronary syndrome (serum levels of high-sensitivity cardiac troponin Ic above the 99th percentile for the normal population, with newonset abnormal features on the electrocardiogram or echocardiogram), a record of acute renal failure (according to the Kidney Disease Improving Global Outcomes guidelines) on the ICU discharge note, documented secondary infection, and the overall length of hospital stay. Vital status and the occurrence of clinical outcomes were last checked for patients who were still hospitalized on May 1, 2020.

Baseline demographics and clinical characteristics were expressed as the median (interquartile range (IQR)) for numerical variables and the frequency (percentage) for categorical variables. Between-group comparisons were performed with the Mann-Whitney-Wilcoxon test (for two groups) or the Kruskal-Wallis test (for more than two groups) for numerical variables and a chi-squared test or Fisher's exact test for categorical variables. A chi-square test for trend was used to determine whether the primary endpoint's frequency of occurrence increased across BMI categories.

Associations between BMI categories and the primary endpoint were probed in a logistic regression analysis. For this analysis, underweight and normal-weight patients were combined into one category $\left(<25 \mathrm{~kg} / \mathrm{m}^{2}\right)$. The results were expressed as the odds ratio (OR) $(95 \%$ confidence interval (CI)). The model's adequacy was checked using the Hosmer and Lemeshow goodness-of-fit test. Multicollinearity was assessed by computing the variance inflation factor. The full-adjusted model included all variables with significant differences when comparing BMI groups on admission.

All statistical tests were two-sided and were performed with R software (version 4.0.0, R Core Team, R Foundation for Statistical Computing, Vienna, Austria). The threshold for statistical significance was set to $P<0.05$.

\section{Results}

From the start of the COVID-19 epidemic up until April 21, 2020, 433 patients with COVID-19 were admitted to Amiens University Hospital. None opposed data collection. Data on BMI were available for 329 of the 433 patients. There were no significant differences between patients with and without available BMI data (Supplementary Table 1). The patients' baseline characteristics are summarized by BMI category in Table 1 . There were 20 patients $(6.1 \%)$ with a BMI $<18.5 \mathrm{~kg} / \mathrm{m}^{2}, 95$ with a normal weight (28.9\%), 90 with overweight (27.4\%), and 124 with obesity (37.7\%). The median age was significantly different between groups $(84.5,81,71$, and 66 years for underweight, normal weight, 


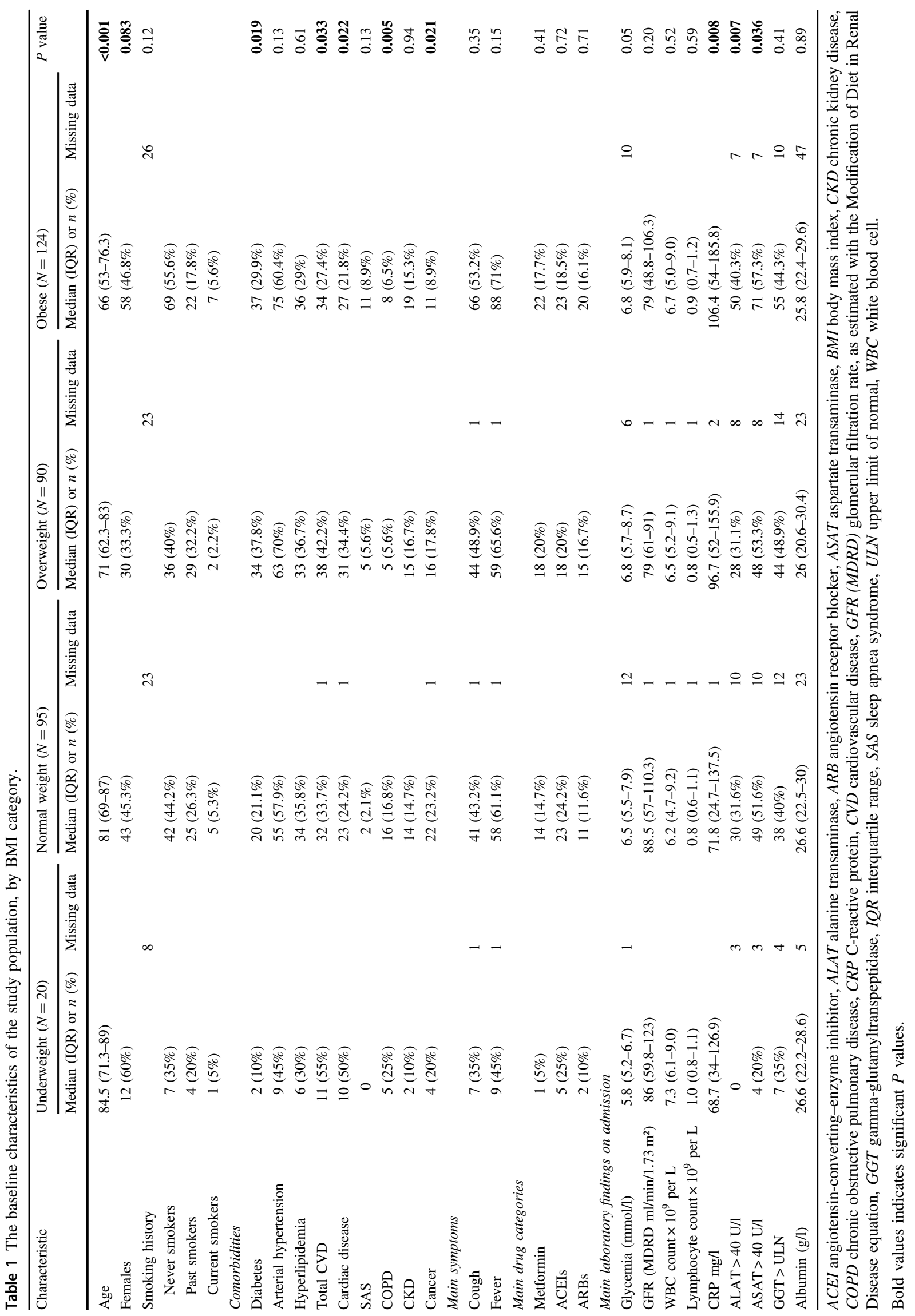


overweight, and obesity, respectively; $P<0.001)$. Interestingly, the higher BMI categories were associated with younger age and a higher C-reactive protein level.

There were significant intergroup differences in the frequency $(\%)$ of patients meeting the primary endpoint during the study period: 7 in the underweight group (35\%), 30 in the normal-weight group (31.6\%), 39 in the overweight group $(43.3 \%)$, and 65 in the obese group $(52.4 \%)$. These results were essentially driven by a higher ICU admission rate with increasing BMI category, whereas the death rate did not differ (Table 2). The prevalence of overweight and obesity among patients admitted to the ICU was $35.1 \%$ and $52.6 \%$, respectively, and the corresponding proportions among those who died were $23 \%$ and $36.1 \%$.

The BMI category was significantly associated with the primary endpoint in a non-adjusted logistic regression $(P=$ 0.006). The respective ORs [95\% CI] for overweight and obesity were $1.61[0.91-2.87]$ and 2.32 [1.38-3.96] $(P=$ 0.0016 for trend). The BMI category was still significant in the fully adjusted model (Supplementary Table 2). The respective ORs [95\% CI] for overweight, and obesity were $1.58[0.77-3.24]$ and $2.58[1.28-5.31]$. The ORs [95\% CI] for ICU admission were, respectively, 5.21 [2.56-11.24] and 6.00 [3.08-12.52] for overweight and obesity in the non-adjusted analysis and 3.16 [1.29-8.06] and 3.05 [1.25-7.82] in the fully adjusted model (Supplementary Table 3$)$. The ORs $[95 \% \mathrm{CI}]$ for death were, respectively, 0.66 [0.32-1.35] and 0.78 [0.41-1.47] for overweight and obesity in the non-adjusted analysis and $1.28[0.50-3.26]$ and $2.89[1.16-7.44]$ in the fully adjusted model (Supplementary Table 4).

The relationship between BMI categories and other endpoints are presented in Supplementary Table 5.

\section{Discussion}

The main findings of our study are as follows. First, 37.7\% of hospitalized patients with COVID-19 were obese, and 27.4\% were overweight. This compares with an estimated prevalence of obesity in Amiens University Hospital's referral area of $\approx 20 \%$ in 2012 [15]. Second, obesity was significantly associated with a greater likelihood of occurrence of the primary endpoint (death or ICU admission), this likelihood was driven by an elevated rate of ICU admission for obese patients. Thirdly, the overweight group had a higher risk (relative to the BMI $<25 \mathrm{~kg} / \mathrm{m}^{2}$ group) of ICU admission but not of meeting the primary endpoint.

The prevalence of obesity in our study was higher than that reported in another study performed in the French city of Lyon (25\%) [16] but was lower than in studies performed in the USA (41.7-48.3\%) [1, 2]. This almost certainly reflects the prevalence of obesity in the background

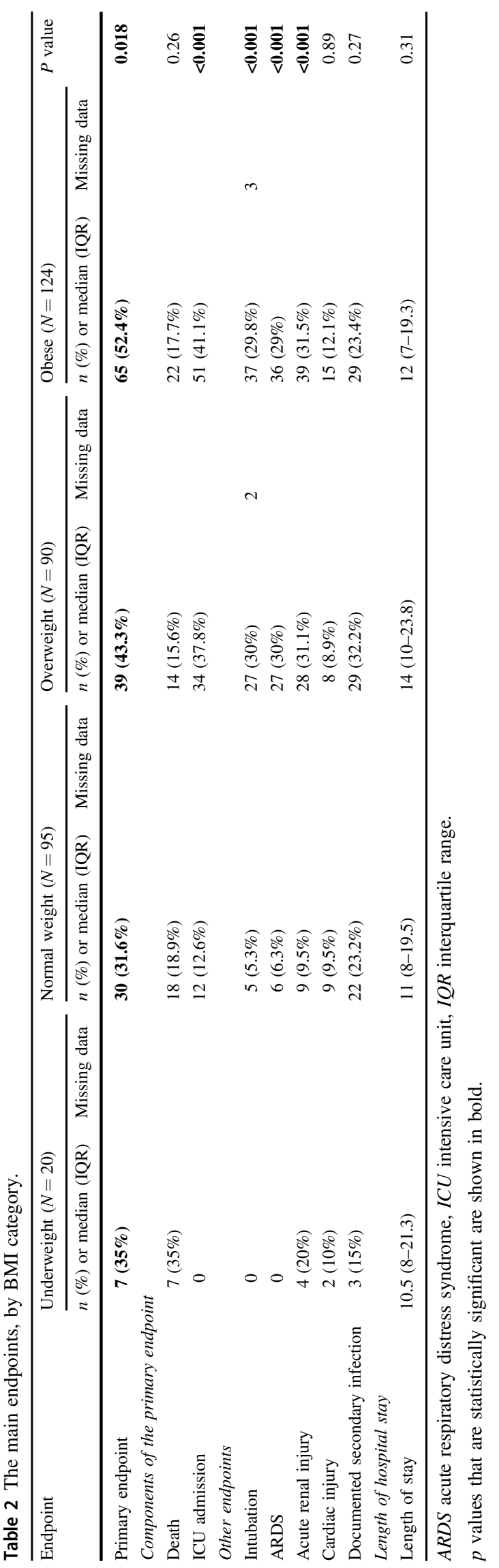


population (12.5\% in the Lyon area in 2012 [15] and 40.2\% in the USA in 2017-2018 [17]). The proportion of obesity among patients admitted to the ICU in our study was similar to those reported for the city of Lille (France [3]) and the UK [18]. However, none of the above-mentioned studies reported the proportions of overweight or underweight individuals, i.e., conditions that might also modify the clinical course of COVID-19.

We found that the frequency of the primary endpoint among patients with COVID-19 was twice as high for obese individuals than for individuals with $\mathrm{BMI}<25 \mathrm{~kg} / \mathrm{m}^{2}$. The relative risk of ICU admission was higher than the risk of the primary endpoint, with a threefold difference in our study, a 2.16-fold difference in the Lyon study, and 6.16 (for BMI $>35 \mathrm{~kg} / \mathrm{m}^{2}$ ) in an American study [19]. Moreover, the relative risk of ICU admission and the incidences of some other endpoints (intubation for mechanical ventilation, ARDS, and acute renal injury) were elevated in the overweight group but without an elevated risk of death suggesting an "obesity survival paradox" phenomenon. This phenomenon has already been reported in patients with pneumonia [11] and ARDS [20]. However, further studies are necessary in order to confirm this phenomenon in the context of COVID-19.

Notably, age was negatively associated with ICU admission but positively associated with death in multivariable analyses. It is probable that younger patients were more frequently referred to the ICU, whereas elder patients were not.

Our study had several limitations-primarily the high proportion of missing data for BMI. However, data on BMI in patients with COVID-19 are scarce [10]. Second, the residual presence of confounding factors cannot be completely ruled out, despite our adjustment for major demographic, clinical, and laboratory variables. Third, other measures of adiposity such as waist circumference were not available.

In conclusion, we found that both overweight and obesity were associated with an unfavorable outcome among patients with COVID-19. Our results need to be confirmed in larger studies and in other populations.

Acknowledgements We are grateful to Edgardo Reyes and Marvin Tchuem Tchuente for their assistance with data extraction.

Funding This research did not receive any specific grants from any funding agencies in the public, commercial, or not-for-profit sector.

Author contributions AAS and JDL designed the study and drafted the paper, which was then revised and approved by all authors; AAS and JDL had full access to all of the data in the study and take responsibility for the integrity of the data and the accuracy of the data analysis. Analysis and interpretation of data: AAS, RD, OG, and JDL. Patient recruitment: JPL, YB, CA, EB, HD, GD, VG, MJ, SL, J Maizel, J Moyet, BV, RD, OG, and JLS.

\section{Compliance with ethical standards}

Conflict of interest The authors declare that they have no conflict of interest.

Publisher's note Springer Nature remains neutral with regard to jurisdictional claims in published maps and institutional affiliations.

\section{References}

1. Garg S, Kim L, Whitaker M, O'Halloran A, Cummings C, Holstein $\mathrm{R}$, et al. Hospitalization rates and characteristics of patients hospitalized with laboratory-confirmed coronavirus disease 2019 COVID-NET, 14 states, March 1-30, 2020. MMWR Morb Mortal Wkly Rep. 2020;69:458-64.

2. Richardson S, Hirsch JS, Narasimhan M, Crawford JM, McGinn $\mathrm{T}$, Davidson KW, et al. Presenting characteristics, comorbidities, and outcomes among 5700 patients hospitalized with COVID-19 in the New York City area. J Am Med Assoc. 2020. https://doi. org/10.1001/jama.2020.6775.

3. Simonnet A, Chetboun M, Poissy J, Raverdy V, Noulette J, Duhamel A, et al. High prevalence of obesity in severe acute respiratory syndrome coronavirus-2 (SARS-CoV-2) requiring invasive mechanical ventilation. Obes Silver Spring Md. 2020. https://doi.org/10.1002/oby.22831.

4. Klang E, Kassim G, Soffer S, Freeman R, Levin MA, Reich DL. Morbid Obesity as an Independent Risk Factor for COVID-19 Mortality in Hospitalized Patients Younger than 50. Obes Silver Spring Md. 2020. https://doi.org/10.1002/oby.22913.

5. Palaiodimos L, Kokkinidis DG, Li W, Karamanis D, Ognibene J, Arora S, et al. Severe obesity, increasing age and male sex are independently associated with worse in-hospital outcomes, and higher in-hospital mortality, in a cohort of patients with COVID19 in the Bronx, New York. Metabolism. 2020;108:154262.

6. Docherty AB, Harrison EM, Green CA, Hardwick HE, Pius R, Norman L, et al. Features of 20133 UK patients in hospital with covid-19 using the ISARIC WHO Clinical Characterisation Protocol: prospective observational cohort study. BMJ. 2020;369:m1985.

7. Lighter J, Phillips M, Hochman S, Sterling S, Johnson D, Francois $\mathrm{F}$, et al. Obesity in patients younger than 60 years is a risk factor for Covid-19 hospital admission. Clin Infect Dis Off Publ Infect Dis Soc Am. 2020. https://doi.org/10.1093/cid/ciaa415.

8. Petrilli CM, Jones SA, Yang J, Rajagopalan H, O'Donnell L, Chernyak Y, et al. Factors associated with hospital admission and critical illness among 5279 people with coronavirus disease 2019 in New York City: prospective cohort study. BMJ. 2020;369: m1966.

9. Williamson EJ, Walker AJ, Bhaskaran K, Bacon S, Bates C, Morton CE, et al. Factors associated with COVID-19-related death using OpenSAFELY. Nature. 2020. https://doi.org/10.1038/ s41586-020-2521-4.

10. Stefan N, Birkenfeld AL, Schulze MB, Ludwig DS. Obesity and impaired metabolic health in patients with COVID-19. Nat Rev Endocrinol. 2020. https://doi.org/10.1038/s41574-020-0364-6.

11. Nie W, Zhang Y, Jee SH, Jung KJ, Li B, Xiu Q. Obesity survival paradox in pneumonia: a meta-analysis. BMC Med. 2014;12:61.

12. Yang X, Yu Y, Xu J, Shu H, Xia J, Liu H, et al. Clinical course and outcomes of critically ill patients with SARS-CoV-2 pneumonia in Wuhan, China: a single-centered, retrospective, observational study. Lancet Respir Med. 2020;8:475-81.

13. Zhou F, Yu T, Du R, Fan G, Liu Y, Liu Z, et al. Clinical course and risk factors for mortality of adult inpatients with COVID-19 in Wuhan, China: a retrospective cohort study. Lancet Lond Engl. 2020;395:1054-62. 
14. ARDS Definition Task Force, Ranieri VM, Rubenfeld GD, Thompson BT, Ferguson ND, Caldwell E, et al. Acute respiratory distress syndrome: the Berlin Definition. J Am Med Assoc. 2012;307:2526-33.

15. Eschwege E, Basdevant A, Charles MA. ObÉpi 2012: enquête épidémiologique nationale sur le surpoids et l'obésité. INSERM/ KANTAR HEALTH/ROCHE. https://www.roche.fr/content/dam/ rochexx/roche-fr/roche_france/fr_FR/doc/obepi_2012.pdf.

16. Caussy C, Pattou F, Wallet F, Simon C, Chalopin S, Telliam C, et al. Prevalence of obesity among adult inpatients with COVID19 in France. Lancet Diabetes Endocrinol. 2020. https://doi.org/ 10.1016/S2213-8587(20)30160-1.
17. Hales CM, Carroll MD, Fryar CD, Ogden CL. Prevalence of Obesity and Severe Obesity Among Adults: United States, 2017-2018. NCHS Data Brief. 2020;8:1-8.

18. Mahase E. Covid-19: most patients require mechanical ventilation in first $24 \mathrm{~h}$ of critical care. BMJ. 2020;368:m1201.

19. Kalligeros M, Shehadeh F, Mylona EK, Benitez G, Beckwith CG, Chan PA, et al. Association of obesity with disease severity among patients with COVID-19. Obes Silver Spring Md. 2020. https://doi.org/10.1002/oby.22859.

20. Zhi G, Xin W, Ying W, Guohong X, Shuying L. 'Obesity Paradox' in acute respiratory distress syndrome: asystematic review and meta-analysis. PloS ONE. 2016;11:e0163677. 